\title{
EL ARGUMENTO SUPERCESIONISTA EN JUSTINO EL FILÓSOFO: EL LÓGOS Y LA HISTORIA DEL CRISTIANISMO
}

\author{
JuLIÁN BARENSTEIN \\ https://orcid.org/0000-0002-3083-7296 \\ Universidad de Buenos Aires \\ Consejo Nacional de Investigaciones Científicas y Técnicas \\ bttp://dx.doi.org/10.15304/ag.39.2.6396
}

\section{Resumen}

Si bien mucho se ha escrito sobre la noción de lógos en Justino, hasta el momento no se la ha estudiado en relación con su concepción de la historia. Aquí nos proponemos poner de manifiesto esa relación descubriendo las premisas del "argumento supercesionista". Por medio de éste, en efecto, es posible recuperar todas las verdades pre-cristianas, especialmente las de origen judaico, y aunarlas en una historia ininterrumpida desde el origen del mundo hasta la primera parousía. Nuestro trabajo se divide, así, en tres partes. En la primera expondremos la noción de lógos, en la segunda examinaremos la reinterpretación de las relaciones entre Judaísmo y Cristianismo en la obra del mártir y en la tercera parte expondremos el argumento supercesionista dando cuenta de cómo opera en ésta la noción de lógos.

Palabras clave: Justino, verdad, lógos, supercesionismo, Cristianismo, apologética.

\begin{abstract}
Although much has been written about the notion of logos in Justin, so far it has not been studied in relation to his conception of history. Here I propose to show that relationship by discovering the premises of the "supercessionist argument". Through it, in fact, it is possible to recover all pre-Christian truths, especially those of Jewish origin, and combine them in an unbroken history from the origin of the world to the first parousia. This paper is, thus, divided into three parts. In the first one, I will expose the notion of logos, in the second
\end{abstract}

Recibido: 06/11/2019. Aceptado: 18/02/2020. 
will examine the reinterpretation of relations between Judaism and Christianity in the work of the martyr, in the third part will show the supercessionist argument explaining how the notion of logos operates in it.

Keywords: Justin, thruth, logos, supercessionism, Christianity, apologetics.

\section{Introducción}

Pretendemos aquí exponer aquél que consideramos el argumento más importante de la apologética cristiana del s. II, y que hemos llamado "supercesionista". Con el término "supercesionismo" se designa usualmente la teología del reemplazo. Bajo este rótulo tan poco transparente se esconde, para decirlo con pocas palabras, una interpretación del Nuevo Testamento que habría sido elaborada por los primeros cristianos $^{1}$ y que se fundaba en la consideración de que la relación actual - y por tanto, nueva- de Dios con los cristianos había reemplazado la que mantenía anteriormente con los judíos, con todo lo que ello implicaba, y esto desde la primera venida de Cristo, la primera parousía, " $\pi \rho \omega ́ \tau \eta ~ \pi \alpha \rho o v ́ \sigma \iota "$, para usar las palabras del propio Justino. Hasta donde nos consta el término habría sido acuñado en inglés y hacia 1870 por Sidney Thelwall, cuando tituló el capítulo tercero de su traducción del opúsculo de Tertuliano, Adversus Iudaeos, precisamente como "On the circumcision and the supercession of the Old Law". En este trabajo, como es obvio, asumimos que existe no ya una teología

${ }^{1}$ A despecho de la diversidad y riqueza del Cristianismo primitivo, inabarcables en este trabajo, cabe aclarar que con "primeros cristianos" en general nos referimos a aquellos que podemos considerar si no ortodoxos, si proto-ortodoxos, entre los cuales contamos, desde ya, a los apologistas, pero no así a los gnósticos cristianos. El lector interesado puede encontrar más información sobre esta tipificación en los excelentes trabajos de David Brakke y Timothy Pettipiece: Brakke, D., The Gnostics. Myth, Ritual and Diversity in Early Christianity, Cambridge-London, Harvard University Press, 2010, esp. cap. I "Imagining Gnosticism and Early Christianities", pp. 1-28; Pettipiece, T., "Variety of Christian Gnosis" en Lössl, J.-Baker-Brian, N. J. (eds.), A Companion to Religion in Late Antiquity, USA, Wiley-Blackwell, 2018, cap. XIV, pp. 297-318. Para un estudio actualizado, aunque parcial, de la posición de Justino ante los gnósticos, nos remitimos a al trabajo de Andrew Hayes: Hayes, A., Justin against Marcion. Defining the Christian Philosophy, Minneapolis, Frotress Press, 2017, esp. pp. 155-160.

${ }^{2}$ Hayes, A., Íbid., pp. 150-160.

El subrayado es nuestro. La así llamada "teología del reemplazo" es mencionada también en otras obras del autor africano, como en el capítulo 21 de su obra más conocida, Apologeticum. Para el estudio del supercesionismo en Tertuliano es insoslayable el trabajo de Geoffery Dunn: Dunn, G. D., Tertullian's Adversus Iudaeos. A rethorical analysis, Washington D. C., The Catholic University of America Press, 2008, esp. cap. 1 "Controversy Surrounding the Text and the Genre", pp. 5-30. 
del supercesionismo, sino un "argumento supercesionista", que es de tenor filosófico-teológico, y que con más o menos precisión y consciencia fue utilizado por todos los apologistas del s. II, tanto los griegos como los latinos. ${ }^{3}$ En Justino, como pretendemos demostrar en el cuerpo de nuestra investigación, este argumento está inextricablemente unido a la noción de lógos que señorea a lo largo de toda su obra, i.e., de la parte que nos ha llegado de ella: ${ }^{4}$ las Apologías I y II (circa 150), y el Diálogo con Trifón (circa 155).

Nuestro planteo se divide, así, en tres secciones de extensión desigual. En la primera, dividida en dos, expondremos, por una parte, la noción de lógos según Justino y, por otra, daremos cuenta de nuestra postura ante la espinosa cuestión del origen de dicha noción. En la segunda, examinaremos un aspecto cenital para la formulación de nuestro punto de vista, a saber, la reinterpretación de las relaciones entre Judaísmo y Cristianismo en la obra del mártir. En la tercera parte, por último, expondremos la versión justiniana del argumento supercesionista dando cuenta de cómo opera en ésta la noción de lógos.

\section{El Lógos, según Justino}

Como sucede con todos los conceptos filosóficos de largo alcance, Justino no expone linealmente su noción de lógos. En la Apología I, y siempre en nuestra interpretación, presenta una primera aproximación que es completada recién en la Apología II y ejemplificada de múltiples maneras en ambas apologías y en el Diálogo con Trifón.

${ }^{3}$ Véase a modo de ejemplo: Arístides, Apología, XIV; Taciano, Discurso contra los Griegos, 29.4-12; Atenágoras, Legación a favor de los cristianos, 7; Teófilo, Tres libros a Autólico, II.30; Hermias, Escarnio de los filósofos paganos, 19; Tertuliano, Adversus iudaeos, 3; Minucio Felix, Octavius, XIX.15 et passim.

${ }^{4}$ Entre las obras perdidas de Justino se cuentan un Discurso contra los griegos ( $\pi \rho \grave{s} \varsigma$

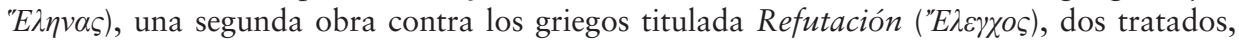

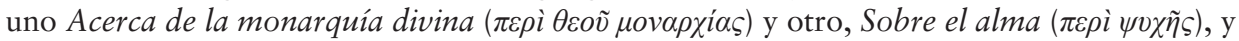
un trabajo titulado El salmista ( $\Psi \alpha \dot{\lambda} \tau \eta \zeta$ ). Los datos aquí señalados provienen de Eusebio (Historia Eclesiástica, 18.3-6). Con gran probabilidad Eusebio no esté mencionando los títulos de las obras perdidas de Justino sino sólo haciendo referencia al contenido de estas. Prueba de

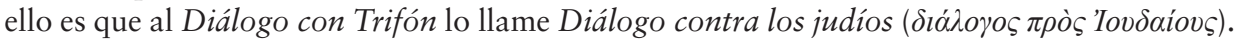

${ }^{5}$ En sentido estricto no se puede hablar de dos apologías. Como es obvio ya en una primera lectura de estos textos, la llamada "Apología II" no es sino un agregado de la Apología I, en el cual amplía y aclara alguno de los puntos expuestos en ésta. Una de las tantas pruebas de esto estriba en que la Apología II no presenta una dedicatoria, tal como es usual

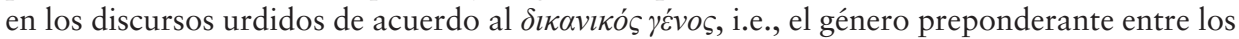
apologistas del siglo segundo (Cf. Apología II, 1). 
El filósofo expone el propósito de la Apología I recién en el capítulo 23, de un total de 68. Se lee allí que su objetivo es presentar pruebas de que lo que afirman los cristianos, es decir, todo lo que han aprendido del propio Cristo y de

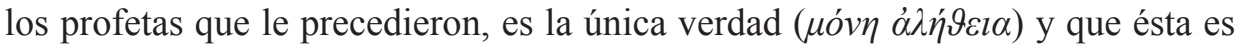
más antigua $(\pi \rho \varepsilon \sigma \beta \delta \tau \varepsilon \rho \alpha)$ que todos los escritores $(\sigma v \gamma \gamma \rho \alpha \phi \varepsilon \tilde{i} \varsigma)$ que han existido. En esta afirmación, Justino asume sin muchas explicaciones que Jesucristo es el

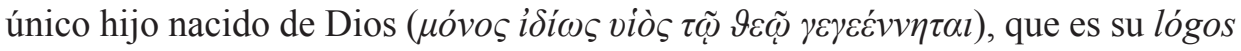

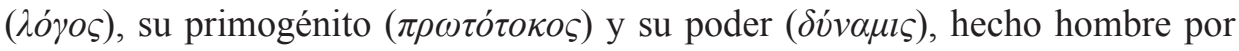

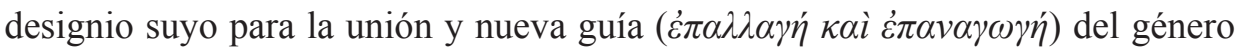
humano, y que si en el tiempo histórico anterior a su encarnación hubo hombres que actuaron y pensaron correctamente no lo hicieron sino guiados por el lógos: ${ }^{6}$

No somos, pues, nosotros <los cristianos $>$ los que profesamos opiniones iguales a los otros, sino que todos, por imitación, repiten nuestras doctrinas. ${ }^{7}$

En este sentido, los pasajes que dan cuenta de su posición son aquellos en los que aborda (a) la cuestión del libre albedrío, noción que contrapone

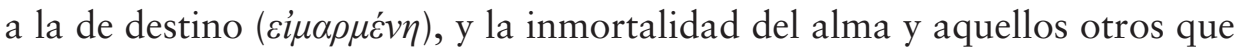
tratan acerca de (b) la Creación del mundo.

En cuanto al primer punto (a), a quienes escogieron el bien - escribe el apologista- les espera una digna recompensa, y a los que hicieron lo contrario, les espera un digno castigo, pues Dios hizo al hombre de modo tal que libremente puede ser otra cosa de lo que es. ${ }^{8}$ La doctrina del libre albedrío, sostiene, ha sido enseñada por el Espíritu Santo profético (ö $\gamma$ ro $\pi \rho o \phi \eta \tau \iota \kappa o ́ \varsigma ~ \pi v \varepsilon \tilde{v} \mu \alpha)$, tal como atestigua Moisés, ${ }^{9}$ al punto que Platón, ${ }^{10}$ la habría tomado del él, que es más antiguo que todos los escritores griegos. Del mismo modo, cuando tanto los filósofos como los poetas griegos hablan sobre la inmortalidad del alma y la contemplación de las cosas celestes, habrían tomado sus afirmaciones de los profetas. De ahí, remata, que parezca haber en todos ellos algo así como los gérmenes o semillas de la verdad $(\sigma \pi \dot{\varepsilon} \rho \mu \alpha \tau \alpha \dot{\alpha} \lambda \eta \vartheta \varepsilon i \alpha \varsigma)$. Se trata sólo de los gérmenes porque una lectura de

${ }^{6}$ Cf. Apología I, 23.1-2.

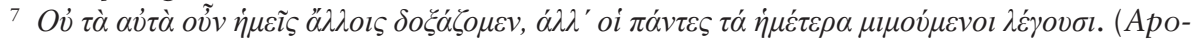
logía I, 60.10) Todas las citas del texto griego de las Apologías I y II proceden de la edición de Nyström (Nyström, D. E., The Apology of Justin Martyr, Tubingen, Mohr Siebeck, 2018).

${ }^{8}$ Cf. Apología I, 43.7.

${ }^{9}$ Cf. Ibid., 44.1.

${ }^{10}$ Justino se refiere explícitamente al pasaje de República 617, donde Platón escribe que "la culpa es de quien elige, Dios no tiene la culpa" (Cf. Apología I, 44.8). 
conjunto de sus escritos revela las múltiples contradicciones entre uno y otro autor, signo inequívoco para Justino de los errores de comprensión. ${ }^{11}$

En cuanto al segundo punto (b), también del lógos que habló por los profetas tomó Platón lo que dijo sobre la Creación del mundo: Dios, había dicho el filósofo ateniense, lo creó transformando una materia informe. ${ }^{12}$ Así, después de comparar las afirmaciones de Platón con los primeros versículos del Génesis en la versión de los LXX, Justino concluye que se sigue de las palabras de Moisés que el mundo fue hecho de elementos preexistentes, que el máximo profeta fue el primero en señalarlo y que Platón y los que siguen sus doctrinas, y los mismos cristianos, lo aprendieron de él. ${ }^{13}$

En la Apología II, de una extensión equivalente a un quinto de la I, y de sólo 15 capítulos, Justino vuelve a la carga. En el texto amplía ciertos argumentos de la Apología I y les da mayor precisión. Ya en el inicio afirma, otra vez, que aquello de bueno que enseñaron los filósofos y los legisladores más virtuosos no fue elaborado por ellos sino por la parte del lógos a la que

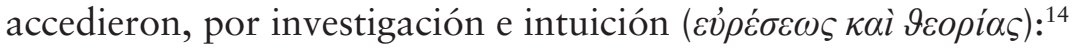

$<$ De $>$ algunos que profesaron la doctrina estoica, sabemos que han sido odiados $[\ldots]$, pues, $[\ldots]$ en los razonamientos éticos se mostraron moderados, al igual que

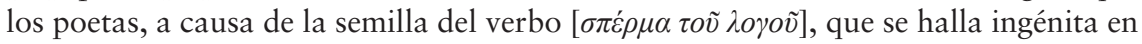
todo el género humano... ${ }^{15}$

En el pasaje se va perfilando la terminología definitiva con la expresión

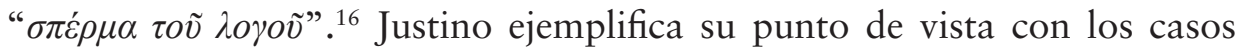

${ }^{11} \mathrm{El}$ argumento de la contradicción en la filosofía de los paganos tomadas como un continuum es recurrente en las obras de Justino y lo encontramos también en las de otros apologistas y autores cristianos de los siglos II-III y, más aun, nuestro filósofo utiliza el mismo argumento de la contradicción en el continuum para atacar las sectarios que, desde su perspectiva, se asumen como baluartes del verdadero Cristianismo, a saber, marcionitas, valentinianos, basilidianos, saturnilianos, etc. cf. Apología I, 44.7-10, Diálogo con Trifón, 2.1 y 35.6-8. También cf. Hermias, Escarnio de los filósofos paganos 2.4 et passim, Clemente de Alejandría, Protréptico, I.

${ }^{12}$ Justino se refiere explícitamente al pasaje de Timeo 36a-c (Cf. Apología I, 59.1; 60.1).

${ }^{13}$ Cf. Apología I, 60.1-5.

${ }^{14}$ Cf. Apología II, 10.2.

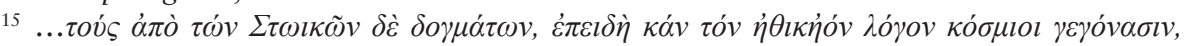

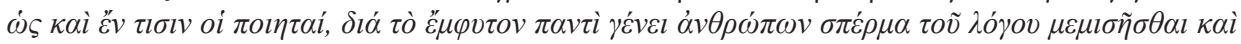

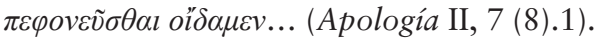

${ }^{16}$ Es dable tener en cuenta que Justino utiliza también, aunque en menor medida, la

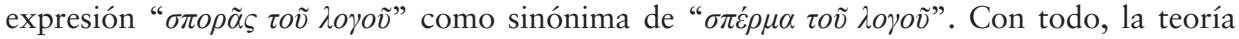
justiniana del lógos seminal ha quedado ligada a esta última denominación. A modo de ejemplo, cf. Apología II, 13.5 . 
de Sócrates, ${ }^{17}$ Heráclito, y Musonio Rufo, ${ }^{18}$ entre los romanos, a la sazón, maestro de Epícteto. El trágico final de estos hombres que vivieron según el lógos antes de la encarnación del lógos, considerados impíos y amigos de las novedades, ${ }^{19}$ se debe, en la interpretación del filósofo, a la acción de los demonios. Éstos incluso, alega con genuino espíritu apologético,

...tratan de hacer odiosos, y con más empeño aun, a los que viven no ya conforme

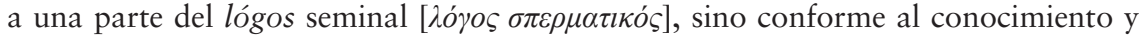

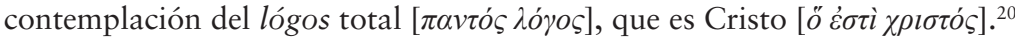

La religión cristiana es más sublime, en suma, que toda la enseñanza humana, por la sencilla razón de que los cristianos poseen el lógos entero

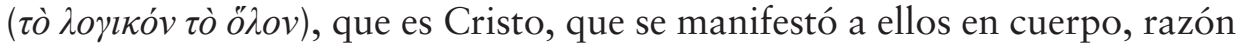

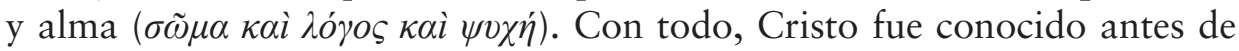
su primera venida $(\pi \rho \omega ́ \tau \eta \pi \alpha \rho o v ́ \sigma \iota \alpha)$, pues, "él era y es el lógos que está en todo, él fue quien por medio de los profetas predijo lo porvenir y quien, una vez hecho de nuestra naturaleza, por sí mismo nos enseño estas cosas." 21

\subsection{Origen de la noción de Lógos en Justino y precisiones estratégicas}

Como es sabido, "lógos" es un término polisémico y de una extensísima tradición en la literatura griega. Pensamos, pues, que dar cuenta del origen de la noción justiniana de lógos es fundamental para comprender su sentido último en la obra del apologista. Ahora bien, mucho se ha escrito acerca de esta noción en Justino y de su procedencia. Para hacer referencia solo a algunas de las contribuciones más destacadas, encontramos, por una parte, caracterizaciones generales, a las que podríamos llamar "manualísticas", como la de Arthur Armstrong hasta las más recientes de Josep Batalla, Claudio Moreschini, Denis Minns y Marian $\operatorname{Hillar}^{22}$ y, por otra, exposicio-

${ }^{17}$ Cf. Apología II, 10.3.

${ }^{18}$ Cf. Ibid., 7 (8).1.

${ }^{19}$ Cf. Ibid., 10.3.

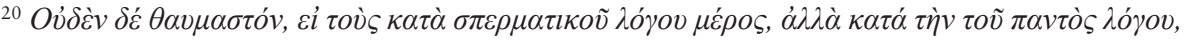

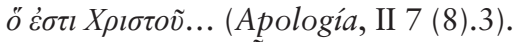

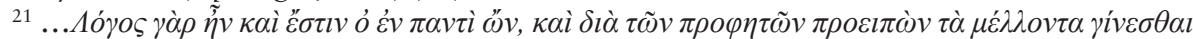

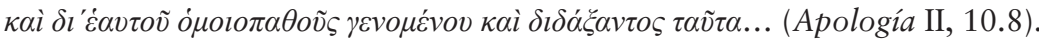

22 cf. Armstrong, A. H., Introducción a la Filosofía Antigua, Buenos Aires, Eudeba, 19938, cap. XIV, pp. 265-269; Batalla, J., “Justí el Filòsof (c. 100-c. 165)” en Font, P. Ll. (coord.), Història del pensament Cristià, Barcelona, FJM, 2002, cap. 3, pp. 53-74; Moreschini, C., Storia della Filosofia Patristica, Brescia, Morcelliana, 2005, esp. pp. 67-69; Moreschini, C., Storia del Pensiero Cristiano Tardo-Antico, Milano, Bompiani, 2013, pp. 245-248; Minns, D., “Justin Martyr” en Gerson, Ll. P., (ed.), The Cambridge History of Philosophy in Late Antiquity, Cambridge, Cambridge University Press, 2010, cap. 14 pp. 258-269; Hillar, M., From Logos to Trinity. Religious Beliefs from Pythagoras to Tertullian, Cambridge, Cambridge University Press, 2012, pp. 138-169. 
nes más detalladas, como las ya clásicas pero insoslayables obras de Erwin Goodenough y Leslie Barnard junto con las más actuales de Eric Osborn y Giuseppe Girgenti. ${ }^{23}$ Con todo, incluso en estos últimos trabajos de largo aliento sobre Justino, la cuestión del origen de su noción de lógos no ocupa más que un capítulo, y no siempre, y las más de las veces no se discute en detalle acerca del origen de la noción, ${ }^{24}$ con las singulares excepciones de Goodenough y Barnard quienes la hacen depender de Filón y del platonismo medio respectivamente. ${ }^{25}$ En términos más puntuales, esa discusión se puede seguir en trabajos más breves, que incluyen una legión de autores. De estos, conviene destacar a Ragnar Holte, Carl Andresen, John Cramer, Johannes Pfättisch, los cuales discuten con Goodenough, y Mark Edwars, ${ }^{26}$ quien discute con todos ellos y, además, con Barnard. Es por esto último que nos interesa reseñar aquí con particular detalle la posición de Edwards. ${ }^{27}$

${ }^{23}$ Goodenough, E. R., The Theology of Justin Martyr, Jena, Verlag Frommannsche Buchhandlund, 1923, cap. V, pp. 139-175; Barnard, L. W., Justin Martyr. His Life and Though, Cambridge, Cambridge University Press, 1967, cap. VII, pp. 85-100; Osborn, E. F., Justin Martyr, Tübingen, Mohr Siebeck, 1973, pp. 28-43; Girgenti, G., Giustino Martire, il primo cristiano platonico, Milano, Vita e Pensiero, 1993, pp. 102ss.

${ }^{24}$ Eric Osborn se detiene en las implicancias teológicas y cristológicas del lógos de Justino antes que en los aspectos filosóficos que dan forma a este. En relación al origen del concepto, Osborn parece estar más inclinado hacia la posición de Goodenough, que la hace dependiente de las elucidaciones acerca del lógos de Filón. Giuseppe Girgenti no se detiene en la noción de lógos, ni mucho menos en su origen, más aun, ni siquiera dedica un capítulo de su libro al tema. (Cf. Osborn, E. F., Justin Martyr..., pp. 28-32; Girgenti, G., Giustino Martire, il primo cristiano platónico..., pp. 7ss).

${ }^{25}$ Cf. Goodenough, E. R., The Theology of Justin Martyr..., p. 175, y Barnard, L. W., Justin Martyr. His Life and Though..., p. 99.

${ }^{26}$ Holte, R., "Logos Spermatikos. Christianity and Ancient Philosophy according to St. Justin's Apologies" en Scripta Theologica 12(1958), pp. 109-168; Andresen, C., "Justin und die mittlere Platonismus" en ZNTW 44-3 (1952), pp. 157-198; Cramer, J. A., "Die Logosstellen in Justinus Apologien kritisch untersucht" en ZNTW 2 (1901), pp. 300-322; Pfättisch, I. M., Der Einfluss Platons auf die Theologie Justins des Martyres. Forschungen zur christliche Literatur und Dogemengeschichte, Paderborn, 1910; Edwards, M. J., "Justin's Logos and the Word of God" en Journal of Early Christian Studies 3-3 (1995), pp. 261-280.

${ }^{27}$ Hemos estudiado otros artículos acerca del lógos en Justino, los cuales no podemos mencionar en el cuerpo de nuestro trabajo por cuestiones de relevancia. Tenemos, pues, por una parte los siguiente trabajos, de Manuel De Almeida Trindade y José Da cruz Lopes Marques: De Almeida Trindade, "S. Justino -a sua doutrina sobre o Logos" en Medievalia. Textos e Estudos 7-8 (1995), pp. 79-97; Da Cruz Lopes Marques, J., "Logos seminal e Logos total na Apologia de Justino, o Mártir” en Revista Caminhando v-21 (2016), pp. 209-223. Escritos ambos en portugués, estos artículos presentan algunas concepciones generales acerca del concepto justiniano de lógos, sin entrar en el detalle acerca del origen de la noción, ni de las discusiones eruditas acerca del tema de Holte, Andresen, Goodenough, Cramer, Pfättisch, Barnard, ni mucho menos, de Edwards. El trabajo de Da Cruz Lopes Marques, p.e., no 
En un artículo publicado a mediados de los 90` , $^{28}$ Edwards sostenía, a diferencia de la mayoría de los estudiosos del filósofo mártir (en especial, Andresen, Cramer, Pfättisch y Barnard, quienes se empeñaban en hacer surgir la noción justiniana de lógos de las filosofías estoica o platónica o de ambas, exclusivamente), que era conveniente buscar las raíces de dicha noción en la tradición bíblica. Este enfoque (que se apoya parcialmente en la investigaciones de Goodenough y Holte, y quizá también en las de C. H. Dodd), ${ }^{29}$ sostiene Edwards, no sólo permite establecer la continuidad terminológica y conceptual —en este punto en particular- de las apologías y el Diálogo con Trifón, sino también evita el problema de considerar a Justino como escribiendo siempre para sus rivales, a saber, los paganos en las apologías y los judíos en el Diálogo, tal como sugieren la mayoría de los comentadores, en especial Robert Grant. ${ }^{30}$ Para Edwards, en suma, Justino

considera ni siquiera la cuestión acerca del número de apologías que escribió Justino, pero lo más sobresaliente de estos trabajos es que a juzgar por la bibliografía consignada en ambos, sus respectivos autores no dan cuenta de estar al tanto de la discusión sobre el tema. (Cf. De Almeida Trindade, "S. Justino -a sua docutrina sobre o Logos"..., pp. 96-97 et passim y Da Cruz Lopes Marques, J., "Logos seminal e Logos total na Apologia de Justino, o Mártir”..., p. 223 et passim). Por otra parte, vale la pena tener en cuenta dos artículos, aunque no son tan importante para nuestro tema, a saber, los de Runar Thornsteinsson y Patrick Mwania: Thorsteinsson, R. M., "Justin and stoic cosmo-theology" en The Journal of Theological Studies, New Series, 63-2 (october 2012), pp. 533-571; Mwania, P., “The Justin Martyr's Concept of Logos Spermatikos and its relevance to theological conversation in Africa today" en ROCZNIKI TEOLOGIZNE LXIV-10 (2017), pp. 189-204. Thorsteinsson bucea en las obras de Justino buscando las alusiones a los estoicos y las referencias a la filosofía estoica, para dar cuenta del rechazo del filósofo a algunos elementos básicos de la cosmovisión de esta escuela, como las nociones de Dios y del Destino o "cosmo-teología”, como llama el autor a las dos conjuntamente. Si bien el trabajo es de tenor general, tiene la virtud de presentar un registro de los pasajes clave de las obras de Justino en los que aparecen los conceptos derivados del Estoicismo y, por tanto, de dar cuenta de los vastos conocimientos que el mártir poseía sobre esta filosofía (Cf. Thorsteinsson, R. M., "Justin and stoic cosmo-theology”..., pp. 270-271). El artículo de Mwania, aunque totalmente ajeno a las especulaciones filosóficas que nos interesan, tiene dos méritos, en nuestra opinión. Primero, el de dar cuenta de un aspecto de la noción de lógos, i.e., como lógos spermatikós, de Justino y revelar su vitalidad en la actualidad, aunque en el contexto acotado de África contemporánea. Segundo, el de poner en evidencia la función que Justino adjudicaba al lógos spermátikos en sus obras, i.e., la de oficiar de mediador entre el Cristianismo y otras culturas y religiones (Cf. Mwania, P., "The Justin Martyr's Concept of Logos Spermatikos and its relevance to theological conversation in Africa today"..., esp. pp.191-194).

${ }^{28}$ Ver nota $n^{\circ} 26$.

${ }^{29}$ Cf. Dodd, C., H., Interpretación de Cuarto evangelio, Madrid, Cristiandad, 1978, esp. pp. 279-282.

${ }^{30}$ Cf. Grant, R. M., "Five Apologistis and Marcus Aurelius" en Vigiliae Christianae 42 (1988), pp. 1-17. 
no adaptaba su discurso en estas obras a un figurado y cambiante auditorio, sino que escribía siempre para instrucción de la Iglesia. ${ }^{31}$

Aunque desde una perspectiva general la posición de Edwards es correcta y superadora, no nos parece incompatible postular un doble origen de la noción de lógos en Justino. Es más, pensamos que el filósofo utiliza dicha noción en sus argumentaciones con plena conciencia de su ambivalencia y de su utilización por parte de autores paganos y cristianos, i.e., no ignoraba el uso que de ella hacían los estoicos, ${ }^{32}$ los platónicos medios ${ }^{33}$ y algunos filósofos antiguos, como Heráclito, ${ }^{34}$ ni tampoco que esta se podía rastrear en la tradición bíblica tanto judaica ${ }^{35}$ como cristiana, en particular, desde ya, en el evangelio de Juan. ${ }^{36}$

Para decirlo con otras palabras, pensamos que Justino utiliza el término "lógos" de manera estratégica, mientras que carga ese término con un significado sui generis. El uso del término implica, en efecto, una estrategia que nos resulta obvia: la familiaridad de la expresión entre los paganos cultos sienta las bases para una discusión que versa, al menos en apariencia, sobre algunos puntos en común. Otra cuestión es determinar no ya el uso del término "lógos" sino lo que Justino entiende que este significa. Y hemos visto (en 1) que Justino menta dos cosas cuando piensa en el lógos, a saber, (a) la providencia divina, presente bajo la forma de lógos spermátikos en fuentes paganas y judías, y (b) la segunda persona de la Trinidad, el Hijo, el Cristo, accesible a los cristianos como pantós lógos.

${ }^{31}$ Para más detalles, cf. Edwards, M. J., "Justin's Logos and the Word of God” en Journal of Early Christian Studies 3-3 (1995), esp. pp. 270-278; Andresen, C., "Justin und die mittlere Platonismus"..., pp. 193-198; Cramer, J. A., "Die Logosstellen in Justinus Apologien kritisch untersucht”..., pp. 311ss; Pfättisch, I. M., Der Einfluss Platons auf die Theologie Justins des Martyres. Forschungen zur christliche Literatur und Dogemengeschichte..., esp. cap. 2; Barnard, L. W., Justin Martyr. His Life and Though..., esp. cap. VII, pp. 101-110; Goodenough, E. R., The Theology of Justin Martyr..., esp. pp. 147-154; Holte, R., "Logos Spermatikos. Christianity and Ancient Philosophy according to St. Justin's Apologies”..., esp. pp. 143-150. Un punto de vista similar al de Edwards sostiene Runar Thorsteinsson (cf. Thorsteinsson, R. M., "The Literary Genre and Purpose of Justin's Second Apology: A Critical Review with Insights from Ancient Epistolography” en HTR 105-1 (2012), esp. pp. 107-109).

${ }^{32}$ Cf. Apología II, 7 (8).1.

${ }^{33}$ Cf. Diálogo con Trifon, $2.1 ; 2.6 ; 5.1$, et passim.

${ }^{34}$ Cf. Apología I, 46.3; Apología II, 8.1.

${ }^{35}$ Cf. Dodd, C., H., Interpretación de Cuarto evangelio..., pp. 267ss.

${ }^{36}$ Para las relaciones entre las nociones de lógos joanina y heraclítea, ver Dodd, C., H., Interpretación de Cuarto evangelio..., p. 268. 
Es dable decir aquí que de acuerdo con Pfättisch la expresión “lógos spermatikós" provendría del estoicismo. Los estoicos, en efecto, designaban así al principio ígneo presente en todas las cosas: en el marco del panteísmo cosmológico estoico las almas de los hombres tomaban parte de la sustancia del fuego y el lógos spermatikós era concebido en la fisiología estoica como una suerte de aire sutil que fluía entre los cuerpos. ${ }^{37}$ Ahora bien, si, por una parte, el origen de la expresión es estoico, la utilización que hace Justino es más bien platónica. Siguiendo, pues, a Andresen, Cicerón, quien había estudiado con el platónico Antíoco de Ascalona, utiliza la expresión "semina iustitiae" para referirse a una suerte de justicia innata —o mejor, a la Justicia- que está presente en el mundo desde la primera generación de seres humanos. Habría, de este modo, una semejanza en el uso que hace el Arpinate de la expresión "semina iustitiae" y la utilización de "lógos spermatikós" por parte de Justino. ${ }^{38}$ Por su parte, no se nos escapa que Barnard discute, siguiendo al cardenal Daniélou, acerca de los posibles antecedentes judaicos de la noción, teniendo en cuenta de manera especial la exégesis filoniana y la Haggadah. ${ }^{39}$

Basten, pues, estas precisiones sobre la noción justinana de lógos y su origen, pues, seguir por este camino implicaría entrar de lleno en la cristología del apologista. Y esto, por cierto, desviaría completamente el sentido del presente trabajo. ${ }^{40}$ Sólo nos resta decir que ninguno de los especialistas abordados en este apartado hace referencia a la historia o la antigüedad del Cristianismo ni explícitamente a la relación entre esa historia y el lógos, ni mucho menos a la teología del supercesionismo.

\footnotetext{
${ }^{37}$ Cf. Pfättisch, I. M., Der Einfluss Platons auf die Theologie Justins des Martyres. Forschungen zur christliche Literatur und Dogemengeschichte..., p. 104.

${ }^{38}$ Cf. Andresen, C., “Justin und die mittlere Platonismus”..., pp. 192-195.

${ }^{39}$ Cf. Barnard, L. W., Justin Martyr. His Life and Though..., pp. 94-95.

${ }^{40}$ No se nos escapa que Justino utiliza varios términos que intercambia por "lógos", entre ellos "cristo", "Israel”, "Jacob", "protótokos", el cual ya hemos mencionado, "sacerdote", etc. Para una lista de estos términos o nombres del lógos, nos remitimos al ya citado artículo de Helleman (cf. Helleman, W. E., "Justin Martyr and the Logos: an apologetical strategy" en Philosophia Reformata 67 (2002), esp. pp. 137-142; para un resumen de la cuestión, cf. Barnard, L. W., Justin Martyr. His Life and Though..., pp.100ss) El interesado en este tema encontrará una somera guía en el trabajo de Marian Hillar: Hillar, M., From Logos to Trinity. Religious Beliefs from Pythagoras to Tertullian..., pp. 158-160.
} 


\section{Los judíos y los cristianos}

A Justino no se le escapó que una concepción del lógos semejante a la expuesta conllevaba múltiples problemas. El primero y más apremiante, en nuestra opinión, es el de establecer la correcta relación entre Judaísmo y Cristianismo. Hasta acá tenemos, pues, que el escritor más antiguo es Moisés, y que todo lo verdadero que hay en los escritos de los paganos fue tomado, en sentido amplio, del máximo profeta, aunque en sus textos no fuera completamente claro. Así las cosas, podemos preguntarnos si el que los paganos hayan encontrado algunas verdades se debe a la presencia ininterrumpida del lógos en la historia humana aunque tan sólo en su aspecto

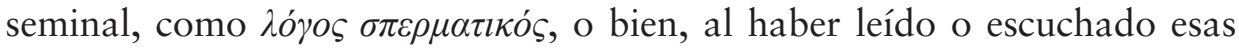
verdades después de haber sido expuestas, por primera vez, por Moisés. No es fácil tomar una decisión. Si optamos por la primera opción, en efecto, la mención de Moisés como el escritor más antiguo no tiene razón de ser, pues el lógos solo con su perenne presencia, aunque seminal, aseguraría el acceso a esas verdades para los espíritus más avisados. Si optamos por la segunda, debemos distinguir el tipo de verdades a las que accedió Moisés y aquellas a las que accedieron los paganos, pues, la propuesta de Justino, aunque él no lo diga expresamente, nos lleva a la conclusión de que mientras los paganos accedieron sólo a los gérmenes de la verdad ( $\sigma \pi \varepsilon \dot{\varepsilon} \rho \mu \alpha \tau \alpha \dot{\alpha} \lambda \eta \vartheta \varepsilon i \alpha \varsigma)$, a través

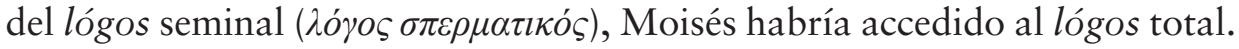
Pero si esto es así, entonces qué sentido tendría la primera parousía, al menos desde la perspectiva del conocimiento. Podríamos matizar la cuestión alegando, ad hoc, que Moisés y con él los profetas no accedieron al lógos total sino sólo una porción superior del seminal que aquella a la que accedieron los paganos, pero Justino nunca se refiere a esta cuestión. ${ }^{41}$

${ }^{41}$ No es ocioso recordar precisamente aquí que Justino tiene un conocimiento tan profundo, aunque no exhaustivo, del Judaísmo y sus variedades que le ha valido el título de “inventor del judaísmo”. En el Diálogo con Trifón, en efecto, el filósofo y mártir utiliza este conocimiento como punto de partida para dar cuenta de la verdad del Cristianismo transformando las profecías del Antiguo Testamento en verdaderas demostraciones proféticas si bien de sabor más retórico que filosófico. A modo de ejemplo, véase Diálogo con Trifón, 49.6, 58.1, 61.1-5, 110.5, 115.6, y 135.3. Para un estudio del conocimiento del Judaísmo en Justino, nos remitimos a los siguientes trabajos: Barnard, L. W., Justin Martyr. His Life and Though..., cap. IV, pp. 39-52; Higgins, A. J. B., "Jewish Messianic Belief in Justin Martyr's "Dialogue with Trypho"” en Novum Testamentum 9- 4 (Oct. 1967), pp. 298-305; Bokser, B. Z., "Justin Martyr and the Jews" en The Jewish Quarterly Review, New Series, 64-3 (Jan. 1974), pp. 204-211; Hirshman, M., "Polemic Literary Units in the Classical Midrashim and Justin Martyr's Dialogue with Trypho" en The Jewish Quarterly Review 83-3/4 (Jan.-Apr. 1993), pp. 369-384; Boyarin, D., "Justin Martyr Invents Judaism” en Church History 70-3 
El segundo problema, que es un derivado del anterior e íntimamente a éste ligado, es el de la existencia de cristianos antes de Cristo, enunciada explícitamente por el apologista. ${ }^{42}$ La cuestión es así: si hubo cristianos antes de Cristo significa que de algún modo se trata de gentes que accedieron al lógos total, antes de que el lógos se encarnara. Aun cuando estos cristianos antes de Cristo lo fueran sola y únicamente desde el punto de vista ético y no desde el cognoscitivo, nuevamente se impone el mismo interrogante: ¿Qué sentido tendría la primera parousía? ${ }^{43}$

Las respuestas, siempre en nuestra opinión, las esboza Justino en el Diálogo con Trifón. Allí, esforzándose por imitar la espontaneidad de los diálogos platónicos, en los que, como al pasar se encuentran los protagonistas, escribe que el judío Trifón se cruzó con él en Éfeso, polo cultural del Asia menor, al término de la Guerra de los Judíos, hacia el $135 .{ }^{44}$ Desde el comienzo el apologista echa mano de la prosopopéia y se figura a su interlocutor como alguien que tiene por costumbre escuchar los discursos de los filósofos. ${ }^{45}$ Hebreo pero griego de cultura, será Trifón quien sentará las bases para definir la relación entre religión y filosofía, primero, y entre Judaísmo y Cristianismo, después. ${ }^{46}$ Con gran habilidad produce Justino momentos de genuina interacción que no se volverán a ver hasta el Octavio

(Sep. 2001), pp. 427-461; Bobichon, Ph., "Préceptes éternels et loi mosaïque dans le "Dialogue avec Tryphon" de Justin Martyr" en Revue Biblique 111-2 (Avril 2004), pp. 238-254.

${ }^{42}$ Cf. Apología I, 46.2-4,

${ }^{43}$ No se nos escapa que Justino intentó responder este interrogante ya en la Apología I, con todo, sus explicaciones no alcanzan la médula del problema y son, por tanto, insuficientes. A modo de ejemplo véase Apología I, 46.5-6. Por lo demás, el que planteamos aquí es uno de los mayores problemas de la historia teológica cristiana, especialmente si lo juzgamos a partir de su permanencia a lo largo de la historia del Cristianismo y su recurrencia en autores de amplio vuelo especulativo. Para no mencionar una legión de filósofos y teólogos que hicieron hercúleos esfuerzos por solucionarlo basta con tener en cuenta que hacia fines del s. XV Pico della Mirandola se planteó al problema y propuso una solución que sólo podía ser entendida —aunque finalmente no lo fue- desde su horizonte epocal: para el conde mirandolano la clave para comprender la correcta relación entre judíos y cristianos residía en efectuar una interpretación anagógica, i.e., cabalística, del libro del Génesis, interpretación que efectivamente no realizaban los judíos. En su propuesta, Pico seguía de lejos pero expresamente a Justino (Cf. Pico della Mirandola, G., Heptaplus, Expositio tertia. Prooemium et passim y Diálogo con Trifón, 11.4, 19.5, 29.1-3, 125.1-5, 130.1-3 et passim).

${ }^{44}$ El encuentro de Justino con Trifón y sus impresiones habría sido revivido por el apologista entre el 150 y 155, cuando tras haber escrito las dos apologías de la nueva religión, ponga por escrito los detalles de ese ya lejano encuentro en el Diálogo con Trifón.

${ }^{45}$ Cf. Diálogo con Trifón, 1.1.

${ }^{46}$ Cf. Ibid., 1.3. 
de Minucio Felix y los diálogos de Casisíaco de Agustín. ${ }^{47}$ Estos momentos son propiciados por la concepción de la filosofía como búsqueda de la verdad que señorea todo el texto. ${ }^{48}$

Así, en el Diálogo, que parece dirigido a un público cristiano, ${ }^{49}$ Justino afronta el arduo trabajo no de refutar punto por punto la religión hebrea, tal como se ha sostenido, ${ }^{50}$ sino el de de expurgar el Cristianismo de los vestigios de Judaísmo. Los intercambios, pues, están preñados de un carácter recriminatorio en el que la posición del judío Trifón, es presentada metafóricamente como parte de la dinámica interna del Cristianismo, en un juego donde las doctrinas judía y cristiana pueden entenderse en términos de la otra, es decir, a través de una mutua comparación y distinción. ${ }^{51}$ En repetidas ocasiones, en efecto, se afirma que los cristianos son no sólo los herederos del legado judío, sino su recambio y compleción. Refiriéndose al Antiguo Testamento, p.e., Justino escribe:

....he leído $[\ldots]$ que había de venir una ley última y un testamento principal sobre todos los hombres que aspiren a la herencia de Dios, porque la ley dada sobre el monte Horeb es ya vieja y les atañe solamente a ustedes <los judíos $>$. Empero la otra pertenece a todos de manera absoluta. Y una ley puesta contra otra ley anula la primera, y un testamento hecho posteriormente, deja igualmente sin efecto el primero. $\mathrm{Y}$ a nosotros Cristo nos ha sido dado como ley eterna y última y como testamento fiel, después del cual ya no hay ni ley, ni ordenación, ni mandamiento. ${ }^{52}$

${ }^{47}$ Véase, p.e., Diálogo con Trifón, 2.1.

${ }^{48}$ Cf. Ibídem.

${ }^{49}$ Según Frances Young, el texto, dirigido a cristianos, representa un desarrollo esencial de la empresa apologética y la elección del diálogo platónico estriba en que está dirigido a gente como él, que aspira a una vida filosófica. Espera, pues, siempre según Young que su auditores encuentren en el diálogo un texto atractivo, fácil de leer y en un estilo al que ya están acostumbrados. (Cf. Young, F., "Greek Apologists of the Second Century" en Edwards, M.-Goodman, M.-Price, S.-Rowland, Chr. (eds.), Apologetics in the Roman Empire, Oxford-New York, Oxford University Press, 1999, pp. 84-85; También cf. Edwards, M. J., “Justin's Logos and the Word of God”..., pp. 278 y 280).

${ }^{50}$ Ver nota anterior.

${ }^{51}$ Estamos de acuerdo con Frances Young porque asumimos que Justino emprende en esta obra no un diálogo verdadero y abierto con los judíos, sino que pretende expurgar el cristianismo del judaísmo. A modo de ejemplo, cf. Diálogo con Trifón, 46.1, 47.2-5.

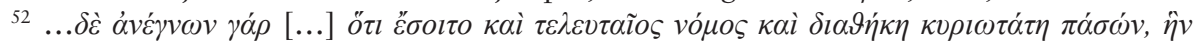

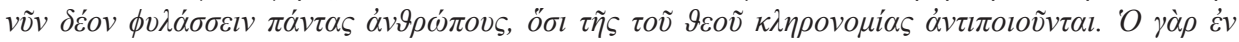

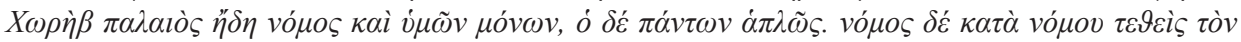

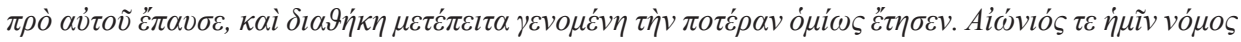

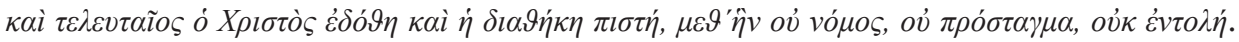
(Diálogo con Trifón, 11.2) Todas las citas del texto griego del Diálogo con Trifón de la edición de Bobichon (Bobichon, Ph., Dialogue avec Tryphon. Édition critique, traduction, commentarire, Fribourg, Academic Press, 2003, 2 vols.). 
Para decirlo con otras palabras, la justificación del Cristianismo, es decir, su verdad, descansa bajo este punto de vista en las promesas del Antiguo Testamento, cuya antigüedad, por lo demás, se remonta, siempre en esta línea de argumentación, a tiempos anteriores a la época homérica, y podría operar como fuente, además, de la literatura pagana producida entre esa época y el presente del apologista. Es dable destacar que en su exposición Justino avanza desde algunas consideraciones generales sobre la ley mosaica a los libros proféticos, pasando por los oráculos sibilinos, para desembocar, como en el cauce natural de un río, en las enseñanzas del Evangelio. ${ }^{53}$ La barca, sin embargo, sobre la que realiza todo el recorrido es la filosofía, que adquiere ante estas declaraciones el carácter de via regia. El valor, pues, de la filosofía, altísimo por cierto, reside -tal como hemos dicho- en que esta permite la búsqueda de la verdad; búsqueda que, huelga aclararlo, finaliza con la posesión del lógos total en el Cristianismo, que deviene, así, no una religión sino una filosofía, aunque divina ( $\vartheta \varepsilon i ́ \alpha \phi i \lambda o \sigma o \phi i ́ \alpha),{ }^{54}$ superior, en tanto tal, a toda filosofía humana ( $\phi \lambda \lambda o \sigma o \phi i ́ \alpha$ ỏ $v \vartheta \rho \omega \pi \varepsilon i ́ o v) .{ }^{55}$ El detalle es importante toda vez que la filosofía es para Justino, el mayor de los bienes, el más precioso ante Dios, pues ella es la única que conduce hacia Él ${ }^{56}$ y habla, pues, desde su propia experiencia. ${ }^{57}$ Los hombres amados de Dios — explica- que vistieron el hábito de filósofos fueron precedidos por los profetas. ${ }^{58}$ "Filósofo" y "profeta" son, así, términos que refieren a individuos que se ocupan de lo mismo. Tan solo en esto se diferencia el profeta del filósofo: en que el primero se mueve sobre el terreno de la verdad revelada, donde todas las pruebas se remiten en última instancia a la fe y a los milagros, mientras que el segundo debe siempre razonar y efectuar demostraciones..$^{59}$

${ }^{53}$ En cuanto al despliegue interno del Diálogo, desde las controversias más amplias hasta los puntos en litigio más insignificantes se resuelven siempre con un recurso a la anterioridad. Así pues, si hay que convencer a un judío, Trifón, de la verdad del Cristianismo, hay que demostrar que éste procede del Judaísmo, aunque más no sea proféticamente. Lo mismo se observa respecto de la relación entre la cultura y la filosofía pagana y la religión cristiana; todo el problema reside, más que en demostrar, en mostrar que los mitos griegos, no son sino una reescritura de las historias veterotestamentarias, como el mito de Deucalión, cuya fuente sería la historia de Noé (Cf. Apología II, 6 (7).2). Para más detalles sobre el procedimiento general que Justino despliega en el Diálogo, cf. Rajak, T., "Talking at Trypho: Christian Apologetic as Anti-Judaism in Justin's Dialogue with Trypho the Jew", en Edwards, M.Goodman, M.-Price, S.-Rowland, Chr. (eds.), Apologetics in Roman Empire..., pp. 71-80.

${ }^{54}$ Apología II, 12.5 .

${ }^{55}$ Ibid., 15.3.

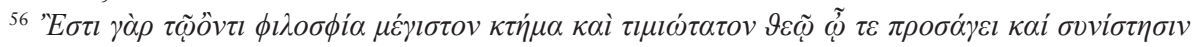
$\dot{\eta} \mu \tilde{\alpha} \varsigma \mu o ́ v \eta . . . .($ Diálogo con Trifón, 2, 1).

${ }^{57}$ Cf. Diálogo con Trifón, 2.1-8.2.

${ }^{58}$ Cf. Ibíd., 7.1.

${ }^{59}$ Cf. Ibid., 7.2. 


\section{El sentido del "supercesionismo"}

Moisés y con él los demás profetas de Israel, en especial Isaías, conocieron, entonces, del lógos aquello que les fue revelado y lo expresaron. Si bien no lo podemos afirmar categóricamente, parecería, como ya sugerimos, no tratarse del lógos total, sino de las semillas del lógos. Con todo, es indudable que estos santos varones pueden pasar a engrosar la lista de los cristianos antes de Cristo, junto con toda una serie de paganos entre los que Sócrates ocupa un lugar de preferencia. Empero para llegar al punto culminante de la argumentación de Justino nos falta aun un paso más, el cual ha quedado contenido y sintetizado en un pasaje inicial del Diálogo que hará historia:

Nosotros <los cristianos> somos el verdadero pueblo de Israel, el espiritual, la raza de Judá, de Jacob, de Isaac y de Abraham... ${ }^{60}$

Como es evidente, Justino distingue entre un Israel espiritual, el verdadero, representado por los cristianos, y un Israel que por comodidad podemos llamar "histórico", representado por los judíos. El primero habría sido anunciado desde el principio de los tiempos ya por el máximo profeta, Moisés, autor de los primeros escritos de la humanidad y aun conservados, y a instancias del lógos. El segundo Israel es el que se puede encontrar en la historia del pueblo judío, contenida también en los libros de Moisés. Llegados a este punto debemos advertir algo que es obvio, a saber, a la luz de las argumentaciones del apologista se vuelve imprescindible para los cristianos efectuar una lectura no literal del Antiguo Testamento. ${ }^{61}$ Es así que si no antes, al menos desde Justino en adelante, los judíos serán acusados precisamente de leer la Torá de manera literal, hasta hoy. ${ }^{62}$

Es evidente, pues, que desde la perspectiva de Justino el lógos siempre existió, siempre estuvo presente y siempre fue conocido, pero no siempre de manera completa sino en su forma seminal. Sólo los cristianos poseen el lógos completo. Ahora bien, si paganos y judíos conocieron el lógos seminal,

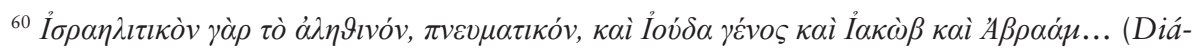
logo con Trifón, 11.5).

${ }^{61}$ Sobre este punto, ver Bates, M. W., "Justin martyr's logocentric hermeneutical transformation of Isaiah's vision of the nations" en The Journal of Theological Studies, New Series, 60-2 (october 2009), esp. pp. 540-545.

${ }^{62}$ No se nos escapa que Filón llevó a cabo una lectura alegórica de las Escrituras, con todo las acusaciones de literalidad recaerán sobre los judíos una y otra vez a lo largo de la historia. A modo de ejemplo, véase, Abelardo, Dialogus inter philosophum, indaeum et christianum, 171. 
no lo hicieron de la misma manera. Los primeros, no tuvieron conciencia de ello, aunque muchos llegaran a morir por él. Los segundos sí, puesto que desde el punto de vista histórico el lógos les anunciaba, a través de los profetas, la compleción de su propia religión. Con todo, la presencia del lógos en la historia humana no tiene su razón de ser sino en la parousía o, mejor,

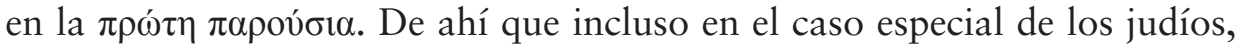
pueblo de Dios, a los ojos del lógos total, del lógos encarnado, de Cristo, en suma, se tornan idénticos a los paganos. Los cristianos son, así, el verdadero Israel, el espiritual. Y, para decirlo con Aristóteles, si el Cristianismo es cronológicamente posterior al Judaísmo, desde el punto de vista lógico - y podemos utilizar el adjetivo "lógico" con un sentido exaltado- es anterior. Podríamos, así, decir que el Cristianismo, entonces, supercede al Judaísmo - y también a la cultura pagana, que sería posterior- y no puede no hacerlo, pues, es, en realidad, el verdadero Judaísmo. Y como Moisés es el primer escritor y el primer profeta, del que salen por vez primera hasta los más mínimos vestigios de la verdad ingénita, la historia humana toda es, de este modo y en el fondo, cristiana de principio a fin.

\section{Conclusión}

Llegados a este punto podemos afirmar, pues, que si la teología del supercesionismo, la teología del reemplazo, está presente en las obras de muchos cristianos primitivos, no ocurre lo mismo con el argumento supercesionista, que aparece recién en la obra de Justino. En este trabajo, tal como habíamos anunciado en la introducción, expusimos las premisas del argumento de acuerdo con la versión de este que nos da Justino, todo lo cual puede ser resumido así: 1) el lógos es la verdad y es eterno, 2) toda verdad histórica hasta la primera parousía es parte del lógos, sus semillas, el lógos spermatikós, y 3) sólo los cristianos poseen el lógos total, el pantós lógos, la verdad completa y eterna. Para Justino toda verdad adquiere su pleno sentido y tiene su razón de ser en la encarnación del lógos, que opera como causa final de un despliegue histórico que hace de toda la Historia, historia de la salvación. Ahora bien, en aquellos de sus trabajos que nos han llegado, todos de marcada impronta apologética, Justino utiliza el argumento para convencer a sus interlocutores. Se vale, pues, de la ambivalencia del término "lógos" para, después de tomarlo como punto de partida, reelaborar su significado, desviándolo de su sentido originario. Asumimos que así procede porque, a soslayo del punto de vista de Edwards, pensamos que Justino no escribe para instrucción de la Iglesia o, cuanto menos, no sólo para ello. 


\section{Bibliografía}

Andresen, C., "Justin und die mittlere Platonismus" en ZNTW 44-3 (1952), pp. 157-198. https://doi.org/10.1515/zntw.1953.44.1.157

Armstrong, A. H., Introducción a la Filosofía Antigua, Buenos Aires, Eudeba, $1993^{8}$.

Barnard, L. W., Justin Martyr. His Life and Though, Cambridge, Cambridge University Press, 1967.

Batalla, J., "Justí el Filòsof (c. 100-c. 165)" en Font, P. Ll. (coord.), Història del pensament Cristià, Barcelona, FJM, 2002, cap. 3, pp. 53-74.

Bates, M. W., "Justin martyr's logocentric hermeneutical transformation of Isaiah's vision of the nations" en The Journal of Theological Studies, New Series, 60-2 (october 2009), pp. 538-555. https://doi.org/10.1093/ its/flp113

Bobichon, Ph., Dialogue avec Tryphon. Édition critique, traduction, commentarire, Fribourg, Academic Press, 2003, 2 vols.

Bobichon, Ph., "Préceptes éternels et loi mosaïque dans le «Dialogue avec Tryphon» de Justin Martyr" en Revue Biblique 111-2 (avril 2004), pp. 238-254.

Bokser, B. Z., "Justin Martyr and the Jews" en The Jewish Quarterly Review, New Series, 64-3 (Jan. 1974), pp. 204-211. https://doi. org/10.2307/1454130

Boyarin, D., "Justin Martyr Invents Judaism" en Church History 70-3 (Sep. 2001), pp. 427-461. https://doi.org/10.2307/3654497

Brakke, D., The Gnostics. Myth, Ritual and Diversity in Early Christianity, Cambridge-London, Harvard University Press, 2010. https://doi.org/10.2307/j.ctvinrvhh

Cramer, J. A., "Die Logosstellen in Justinus Apologien kritisch untersucht" en ZNTW 2 (1901), pp. 300-322. https://doi.org/10.1515/ zntw.1901.2.1.300

Da Cruz Lopes Marques, J. "Logos seminal e Logos total na Apologia de Justino, o Mártir" en Revista Caminhando 21- 2, (jul./dez. 2016), pp. 209-223. https://doi.org/10.15603/2176-3828/caminhando.v21n2p209-223

De Almeida Trindade, M., "S. Justino-A sua doutrina sobre o Lógos" en Medievalia. Textos e estudos 7-8 (1995), pp. 79-97.

Dodd, C., H., Interpretación de Cuarto evangelio, Madrid, Cristiandad, 1978.

Dunn, G. D., Tertullian's Adversus Iudaeos. A rethorical analysis, Washington D. C., The Catholic University of America Press, 2008. 
Edwards, M. J., “Justin's Logos and the Word of God” en Journal of Early Christian Studies 3-3 (1995), pp. 261-280. https://doi.org/10.1353/ earl.0.0039

Girgenti, G., Giustino Martire, il primo cristiano platonico, Milano, Vita e Pensiero, 1993.

Grant, R. M., "Five Apologistis and Marcus Aurelius" en Vigiliae Christianae 42 (1988), pp. 1-17. https://doi.org/10.1163/157007288X00282

Goodenough, E. R., The Theology of Justin Martyr, Jena, Verlag Frommannsche Buchhandlund, 1923.

Hayes, A., Justin against Marcion. Defining the Christian Philosophy, Minneapolis, Frotress Press, 2017. https://doi.org/10.2307/j.ctt1kgqtxg

Helleman, W. E., "Justin martyr and the "logos": an apologetical strategy" en Philosophia Reformata 67-2 (2002), pp. 128-147. https://doi. org/10.1163/22116117-90000259

Higgins, A. J. B., "Jewish Messianic Belief in Justin Martyr's "Dialogue with Trypho"” en

Novum Testamentum 9- 4 (Oct. 1967), pp. 298-305. https://doi. org/10.2307/1560359

Hillar, M., From Logos to Trinity. Religious Beliefs from Pythagoras to Tertullian, Cambridge, Cambridge University Press, 2012. https://doi.org/10.1017/CBO9781139003971

Hirshman, M., "Polemic Literary Units in the Classical Midrashim and Justin Martyr's Dialogue with Trypho" en The Jewish Quarterly Review 83-3/4 (Jan.-Apr. 1993), pp. 369-384. https://doi.org/10.2307/1455159

Holte, R., "Logos Spermatikos. Christianity and Ancient Philosophy according to St. Justin's Apologies" en Scripta Theologica 12(1958), pp. 109-168.

https://doi.org/10.1080/00393385808599790

Minns, D., "Justin Martyr" en Gerson, Ll. P., (ed.), The Cambridge History of Philosophy in Late Antiquity, Cambridge, Cambridge University Press, 2010, cap. 14 pp. 258-269. https://doi.org/10.1017/ CHOL9780521764407.018

Moreschini, C., Storia della Filosofia Patristica, Brescia, Morcelliana, 2005.

Moreschini, C., Storia del Pensiero Cristiano Tardo-Antico, Milano, Bompiani, 2013.

Mwania, P., "The Justin Martyr's Concept of Logos Spermatikos and its relevance to theological conversation in Africa today" en ROCZNIKI TEOLOGIZNE LXIV-10 (2017), pp. 189-204. https://doi. org/10.18290/rt.2017.64.10-14 
Nyström, D. E., The Apology of Justin Martyr, Tubingen, Mohr Siebeck, 2018.

https://doi.org/10.1628/978-3-16-155762-0

Osborn, E. F., Justin Martyr, Tübingen, Mohr Siebeck, 1973.

Pettipiece, T., "Variety of Christian Gnosis" en Lössl, J.-Baker-Brian, N. J. (eds.), A Companion to Religion in Late Antiquity, USA, Wiley-Blackwell, 2018, cap. XIV, pp. 297-318. https://doi.org/10.1002/9781118968130. $\underline{\operatorname{ch} 14}$

Pfättisch, I. M., Der Einfluss Platons auf die Theologie Justins des Martyres. Forschungen zur christliche Literatur und Dogemengeschichte, Paderborn, 1910.

Rajak, T., "Talking at Trypho: Christian Apologetic as Anti-Judaism in Justin's Dialogue with Trypho the Jew", en Edwards, M.-Goodman, M.-Price, S.-Rowland, Chr. (eds.), Apologetics in the Roman Empire, Oxford-New York, Oxford University Press, 1999, pp. 71-80.

Sikora, J. J., "Philosophy and christian wisdom according to saint Justin martyr" en Franciscan Studies 23 (1963), pp. 244-256.

Thorsteinsson, R. M., "The Literary Genre and Purpose of Justin's “Second Apology": A Critical Review with Insights from Ancient Epistolography" en The Harvard Theological Review 105- 1 (january 2012), pp. 91-114.

https://doi.org/10.1017/S001781601100054X

Thorsteinsson, R. M., "Justin and stoic cosmo-theology" en The Journal of Theological Studies, New Series, 63-2 (october 2012), pp. 533-571. https://doi.org/10.1093/jts/fls101

Young, F., "Greek Apologists of the Second Century" en Edwards, M.Goodman, M.-Price, S.-Rowland, Chr. (eds.), Apologetics in the Roman Empire, Oxford-New York, Oxford University Press, 1999, pp. 81-104. 